\title{
Carotenoids Concentration Detection Investigation: A Review of Current Status and Future Trend
}

\author{
Rosdina Rahiman, Mohd Alauddin Mohd Ali, and Mohammad Syuhaimi Ab-Rahman
}

\begin{abstract}
Carotenoids as natural pigment are the class of hydrocarbon and may easily found in vegetables and fruits as well as in human tissue. In human, carotenoids beneficial in health factor as provitamin A, agent of antioxidant, preventing from AMD (age-macular degenartion) in eye disease, reduce risk of prostate cancer, and other high risk disease. Generally, plasma carotenoid appear to be a good indicator and benchmark in measuring total antioxidant status in human using gold standard method, high pressure liquid chromatography, HPLC. Other carotenoids detection area such as macular pigment and skin also give high correlation and significant with plasma carotenoids. Recently, carotenoids detection seem to be more preferrable in animals such as marine life, mammals, non-mammals and birds and as well as in plant. Current status and future trend or direction of detection of carotenoids concentration that have been evaluated with some prediction based on publication among epidermiological and other related studies are presented. The important of planning the direction of carotenoids detection in research activities are identified and suggestions are made for the future research in order to enhance role of carotenoids especially in human as promoting in preventing high risk disease as well as other life for maintaning the environment stability and quality.
\end{abstract}

Index Terms - Carotenoids, macula pigment, plasma, skin.

\section{INTRODUCTION}

Highlight The investigations of the carotenoids have appeared as a scientific, discipline and develop rapidly for the study of epidemiology and high risk disease such as cancer or cardiovascular disease. Carotenoids capability in maintaining health have been proven, thus act as antioxidant defense system in human body. As a defense mechanism, carotenoids quench ROS (reactive oxygen species) and protect against harmful effect of free radicals and other reactive molecules.

Any atom or molecule that possesses an unpaired electron may eventually increase the level of chemical reactivity of the molecule in known as free radical. This occur when unpaired electron sometimes take an electron from other molecule, combine with other molecule or relinquish and reattach itself to other molecule, [1]. This is free radical's fondness to react with other molecule and disturb the system

Manuscript received March 20, 2013; revised May 21, 2013.

Rosdina Rahiman and Mohd Alauddin Mohd Ali are with System Design Research Group, Department of Electrical, Electronic \& System Engineering, Universiti Kebangsaan Malaysia, 43600 Bangi Selangor (email: rosdina @ic.utm.my).

Rosdina Rahiman and Mohammad Syuhaimi Ab-Rahman are with Spectrum Technology Research Group, Department of Electrical, Electronic \& System Engineering, Universiti Kebangsaan Malaysia, 43600 Bangi Selangor. stability. In a normal metabolic process, reactive molecules and free radicals are produced. If the radicals increase significantly, it might stimulate harmful to cell damage or DNA mutation which is called as oxidative stress. As a protective boundary system between environment and body system, human skin developed a defense mechanism such as antioxidant, enzymes and vitamins which able to remove intermediate peroxyl radicals by obstructing the chain reaction of lipid peroxide. Carotenoids as anti-oxidant agent are able to fulfill singlet molecular oxygen scavengers [2] and respond with some radicals species [3].

Carotenoids as antioxidant agent cannot be synthesized by the human body itself. By consuming through topical application of fruits and vegetables and supplements rich of carotenoids, the concentration will increased in order to neutralize several attacks of free radicals and maintain a good health.

Epidemiology and other related studies have shown the importance of carotenoids in preventing and their relationship of human high risk diseases include their bioavailability, metabolic activities and reaction of mechanism. Various interest research areas in carotenoids concentration is rapidly increased since development of technology of non-invasive method give another alternative in research activities which offer more accurate, fast and rapid outcome.

\section{A. Carotenoids}

\section{1) Physical and chemicals properties}

Carotenoids are an organic and natural pigments present in plants and other photosynthetic and non photosynthetic organisms such as algae, yeast, bacteria and others but not synthesized in animals or humans. Carotenoids give red, orange and yellow colours in many plants, fruits and flowers.

All carotenoids usually consists of eight $\mathrm{C}_{5}$ isoprenoids units joined head-to-tailed and posses a polyisoprenoid structure by tail-to-tail linkage of two $\mathrm{C}_{20}$ methyl group and open chain or ring structure form the ends of the chain to produce 40-carbon polyene chain of symmetrical molecule. An importance feature is conjugated of double bond system, which provides light absorption chromophore that gives an attractive colour and visible absorption spectrum as a basis guideline for their identification and quantification. Fig. 1 (a) illustrates an example of basic structure of beta carotene as cyclic carotenoids [4].

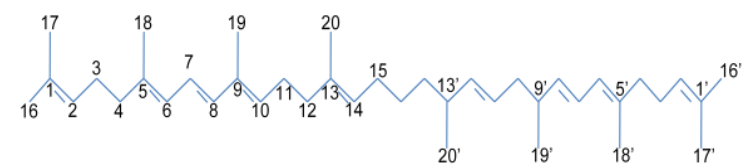

Fig. 1. Basic structure and numbering scheme of beta carotene [4]. 
Carotenoids have been categorized into two parts according to their chemical composition. Oxy-carotenoids or xanthophylls such as lutein and zeaxanthin and non-oxy carotenoids (hydrocarbon carotenoids) or carotene such as beta carotene and lycopene has been identified among more than 600 carotenoids found in natural sources [5]. Carotenoids can be acyclic (e.g. lycopene), monocyclic (e.g. $\gamma$-carotene) or dicyclic (e.g. $\beta$-carotene) and exist naturally in a more stable all-trans form. Carotenoid was transported predominantly by lipoprotein in human plasma. Beta carotene and lycopene are very fat-soluble and inclined to present $75 \%$ in the low-density lipoprotein (LDL) whereas the remaining $25 \%$ of lutein and zeaxanthin that more polar than carotene usually appear in both LDL and high-density lipoprotein (HDL) [5]. Beside fruits and flowers, carotenoids may also be found in some animal species such as birds (swallow, Montagu's harrier), marine life (salmon, red porgy fish) and insects (ladybird beetle).

\section{B. Role of Carotenoid}

Carotenoid pigments have an important role as a protective agent in cells and organisms in nature. Naturally, carotenoids are responsible for determination or contribution of yellow, orange and red colors in fruits and vegetables as well as animals and other live. In plants and algae, carotenoids act fundamental roles as protector against photodamage and contribution in photosynthesis activities.

In human, carotenoids such as beta-carotene and lycopene are able to participate in free radical reactions where they quench or prevent the formation of singlet oxygen through an efficient energy transfer process. In addition, carotenoids act as provitamin A activity, essential pigment for good vision and act as antioxidant. Carotenoids also promote other function in human health such as immuneenhancement, preventing from cardiovascular disease, prevention of macular degeneration, inhibit of cancer and decreased risk of cataract formation, potential increase resistance to pathogens and growing of tumor in early stage [6], [7]. Their physical and chemical properties are summarized in Fig. 2.

Capability of carotenoids in absorbing light is widely known. Typically, absorption values for both beta-carotene and lycopene were occurs at $488 \mathrm{~nm}$ and $514.5 \mathrm{~nm}$ of wavelength respectively using organic solvent during identification of carotenoids in human skin [8]. In order to determine the absorption spectra, $1 \mu \mathrm{g} / \mathrm{ml}$ of chloroform is prepared and obtained the measurement in Lambda 20 (Perkin Elmer) UV/VIS spectrophotometer.

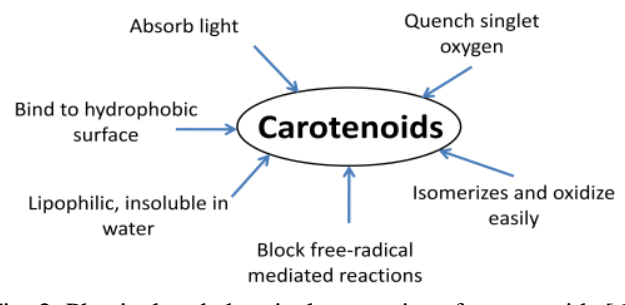

Fig. 2. Physical and chemical properties of carotenoids [6].

After identification of three Raman bands which appear at $1005 \mathrm{~cm}-1,1156 \mathrm{~cm}-1$ and $1523 \mathrm{~cm}-1$, the strong carboncarbon double-bond of $1523 \mathrm{~cm}-1$ Raman lines is selected for detection of beta carotene and lycopene concentration at skin. Transported by blood and located at LDL and HDL, carotenoids mostly found in dermis and epidermis of human skin. Carotenoids also ready and easy to isomerizes and oxidize in the system body and as one of important substances in blocking free-radical reactions because of the capability in quenching singlet oxygen.

\section{TYPES OF DETECTION CAROTENOIDS CONCENTRATION}

Epidemiological studied has introduced types of detection among human in order to measure the total status of carotenoids concentration in human body.

1) Plasma carotenoid

2) Macula pigment of retina

3) Skin or dermal carotenoids.

4) Other life

Generally, carotenoids are insoluble in water and transported in blood by a low-density lipoprotein (LDL) with other fat-soluble substances in human body. This characteristic offers the capability of carotenoid detection through plasma or serum. Via high pressure liquid chromatography (HPLC), status of carotenoid concentration in human body is available. Although carotenoids give a good respond through blood sample of human but when faced with a large amount of tissue and special preparation of fasting blood, determination through blood is not applicable.

Detection through macula pigment of retina gives other alternative although only two substances have been indicate, lutein and zeaxanthin. However, lacks of eye donor give limitation of the study in data collection and data acquisition.

Development of non-invasive technique, open a wide possibilities during measuring carotenoid substances in human body through forehead, palm, knee and others because of carotenoids normally located at sweaty area. However, determination of carotenoids in plant and animal where provide them with colours and helping in absorbing light energy in photosynthesis quite popular among research activities nowadays.

\section{Detection through Plasma Carotenoid}

In early years, detection from serum or known as plasma carotenoids has been used to validate and compare the questionnaire result of long-term carotenoids consumption. As a result it shows that there is a correlation between plasma carotenoids with dietary intake of carotene and vitamin A [9]. Changes in carotenoids concentration have been found through plasma carotenoids for analysis at different dose of carotenoids in a controlled subject. Lowcarotenoid diets of controlled subject using high pressure liquid chromatography (HPLC) technique show a respond with plasma carotenoid in a controlled subject [10]. Plasma carotenoid also has been used to analyze the increasing of carotenoid concentration of high dose dietary of beta carotene in healthy men using a different method such as spectrophotometer which provide more sensitive and reproducible technique [11]. Several studies show that plasma carotenoid concentrations are associated with fruits and vegetable consumptions and act as biomarker tools. In epidemiological studies and nutritional dietary survey, they used blood serum as validation tools to measure the intakes of fruits and vegetables and lifestyle. Thus, plasma 
carotenoid proved as important and useful detection in observing fruits and vegetables intake in human especially in cross-sectional study [12].

The general process of plasma carotenoid is starting from a collection of the blood sample from all plastic syringes or evacuated container. Normally the subject will be asked for normally 8-10 hours or overnight fast before blood sample is collected. They keep the blood sample at a special coated tube to minimize the exposure of carotenoid to the light at a specific range of temperature from $-20^{\circ} \mathrm{C}$ to $-7^{\circ} \mathrm{C}$. After that, separation and quantification process will be done using suitable and efficient technique in order to obtain an accurate and rapidity result. Typically, analysis will be done using HPLC technique which based on the peak areas of HPLC chromatograms.

\section{Detection through Macula Pigment Carotenoid}

Macula pigment (MP) formed of oxy-carotenoids of lutein and zeaxanthin and have higher concentration in macular compared to anywhere else of human body. In 1985, a study has revealed the composition of macula pigment which is similar as a mixture of lutein and zeazanthin [13]. For newborn, it has been found that for prenatal retinas, a yellow spot was never visible. However for postnatal cases, the pigments were noticeable only if their combined masses in the macula exceeded about $5 \mathrm{ng}$. There is also a significant correlation between the lutein concentration in human milk and plasma especially during early lactation in postnatal cases [14]. The levels of carotenoids, retinoids, and tocopherols in normal and cataractous human lenses have been determined and studied. As a conclusion, retinol, retinyl palmitate, and $\alpha$ - and $\gamma$ tocopherols also are present in human lens beside lutein and zeazanthin.

Age related cataract and macular degeneration are the related disease of human retina. Epidemiology studies indicates that age related macular degeneration or AMD occur mostly among elderly population correspond to the low carotenoids concentration in their retina [33]. In 1998, resonance Raman scattering apparatus appear to be a new noninvasive method in measuring carotenoids level in human retina using pigment epithelium of intact frog eyes [15]. With high in sensitivity and specificity, this optical method has utilized and facilitates studies of ocular distribution especially in AMD or other macular disease.

In order to measure total macula pigment carotenoids, normal and reverse HPLC is popular technique among researcher but mass spectrometry technique also give an outstanding result. This technique is able to separate and identify the individual stereoisomer for a various type of carotenoids which analyze for both lutein and zeazanthin by plasma carotenoids. There is an evidence shows that stereoisomer of zeazanthin is not obtain instantly from the diet but cause of chemical processes occurring within the eye [16]. However, there is a consistent hypothesis conclude that by increasing the dietary intake of certain carotenoids foods or people with high concentration of zeaxanthin has a lower the risk of AMD development and modify the macula pigment density [17].

\section{E. Detection through Skin Carotenoid}

The importance of carotenoids in preventing human disease has been well-known among epidemiological and other related studies. In human, carotenoids are found in stratum corneum cell which act as a barrier or boundary against the effects of environment pollutant exposure where it believed to provide antioxidant and photo-protective benefits to the skin [18]. Increases of these substances in blood carotenoids, is then reflected due to the increasing of carotenoids concentration in all organs of human body including skin. As a result, the direct measurement of skin carotenoid seems to provide other alternative in order to gain information about their level or total status of antioxidant carotenoids. Carotenoids pigment has been successfully chemically detect in 1975 by obtaining a skin of non-carotenemic subject at autopsy. The specimens need to be diced, extracted and saponified in a solution of $12 \%$ $\mathrm{KOH}$ in methanol at $40 \mathrm{C}$ for 30 to 60 minute [19]. It also shows that carotenoids present in both dermis and epidermis where carotenoids seem to have high concentration in epidermis compared in dermis.

Beta carotene and lycopene are two dominant carotenoids in skin tissue with backbone of nine conjugated carbons bonds and conjugation into the ionone rings on each end. Normally, the absorption region is in a range from 400nm to $500 \mathrm{~nm}$ wavelength of blue-green spectral. Among other natural carotenoids, lycopene have been traced to be more efficient as oxygen quencher and protective mechanism against UV irradiation on the skin. Beta carotene has ability as a trap or scavenger of peroxyl radicals at low oxygen tension [20].

There are various anatomical skin sites that have been measure with different distribution of carotenoids such as inner forearm, forehead, inner palm, dorsal hand, knee, back of the healthy subject. A suitable skin sites in assessing antioxidant carotenoid level in human skin is the inner palm because it is practical for measuring purpose and also other following reason: a) palm are among the highest carotenoid concentration found in skin (because carotenoids are lipophilic and palm skin has a high lipid/protein ration), b) minimal pigmentation among various skin types c) high in stratum corneum thickness of the palm approximately about 400um compared with other skin sites [21].

Skin antioxidant measurement is not affected by age, gender as well as BMI, carotenoids concentration will only vary by increasing the dietary intake of fruits and vegetables, antioxidant supplements prescribing, thus oxidation stress factor such as smoking or UV light exposure will lower the carotenoids level in human.

\section{F. Detection of Carotenoids in Other Life}

Generally, contribution of carotenoids in photosynthesis, protective agent of photo damage and as membrane stability in plant have been recognized and established in early years. With optimal functioning of photosynthetic pathway, thus leading to gain information on the interaction of carotenoids and light in plant. Nowadays, their attention in botanical area has been shifted to their role in non-human such as animals. In mammals or non-mammals system, carotenoids essentially as pigmentation source. Carotenoids also have capability to increase immune system, available to enhance intercellular communication and act as antioxidant agent. Beside mammal, carotenoids also have been analyzed in birds, marine life and vertebrates. 
A study has been conduct to focus on the pigmentation source of astaxanthin in marine life such as salmon in understanding the dynamics and deposition of carotenoids in this species as a commercial requirement. The study provides a theoretical framework in observing characteristic of an organism as determined by genetic and environmental factors for a quantitative understanding in carotenoids concentration [22]. Although astaxanthin has been shown to improve the red coloration of marine life such as red porgy, other carotenoids sources in promoting yellowish coloration have been investigate. The study shows the ability of Haematococcus algae in promoting the red coloration in red porgy and able to employ variation of dietary in carotenoids. While for yellowish in skin is contributed by spirulina and alfalfa intake during feeding time [23]. However, the protocols for human treatment and utilizing of animal model must be approved by the institution of animal care of the university. Mouse model have been used to identify the carotenoids effect in lung transcriptome and they conclude that mouse present to be a reliable model for bioavailability and bioactivity study [24].

Some plants have an ability to synthesize carotenoids and there are several dozen selection of food that contains carotenoids sources example such as alpha carotene in carrots and red palm oil, beta carotene in apricot, carrot, mango and red pepper, lycopene in pink grapefruit and pink flesh guava, lutein in broccoli and corn, and zeaxanthin in Burrito and corn [25]. In fact, dietary supplementary of carotenoids shows a good relationship in reducing cancer risk by intake of various fruits and vegetables associated with as disease prevention [26].

\section{AsSESSING CAROTENOIDS TECHNIQUE}

Generally, carotenoids assessing technique can be categorized into two types: invasive and non-invasive. Invasive technique prefers a collection of preparation sample before perform or conduct any biopsies or analysis. While, invasive technique is more practical because it did not require any sample during the process of determining and analyzing the substances.

In the past decade, the implementation of gold standard methods for measuring carotenoids using HPLC has been widely used. This technique works very well with plasma carotenoids but facing a problem when measuring using skin because it needs a large amount of tissue. In 1991, HPLC have been used to identify and separate a variety of carotenoids in fruits, vegetables and human plasma such as lutein, zeaxathin, beta-carotene, alpha carotene, lycopene and beta cryptoxanthin [27]. The instrumentation were originally developed for gas chromatography (GC) and liquid chromatography (LC) and first introduced at 1960s. Typically, normal phase chromatography and reverse phase chromatography are two types of HPLC that suits well with carotenoids detection via plasma. However, this technique is a highly invasive, relatively time-consuming and using expensive chemical but as a gold standard achievement, HPLC is generally favored among epidemiology activity as validation method.

Most of carotenoids detection in plasma used HPLC technique, [11], [12]. HPLC also have been widely used during macula pigment of carotenoids detection [13], [15]. This is based on the function of HPLC in separating, identifying and quantifying compounds. As an alternative to HPLC, other technique which involved in carotenoids detection are flicker photometry, scanning laser ophthalmoscope, reflectance spectroscopy and other.

Initially, resonance Raman spectroscopy has been used as optical approach for detection of carotenoids in human retina. Based on previous studies in human retina, it was found that the Raman spectroscopy technique works well for detection of the macular carotenoids pigments after some test and development. As a conclusion, resonance Raman scattering appear to be an outstanding tools as non-invasive optical technique to measure carotenoids level in living human retina,[15], [28], [29]. In addition, this non-invasive technique give high promising outcome, fast, precise, sensitive and quantitative as well as could be used for a patient with a variety in ocular pathologies.

Development in non-invasive Resonance Raman spectroscopy, widen the research interest in epidemiology and particular studies. As an approach for skin carotenoid detection, this technique is favorable among research activities because of their ability to discern carotenoids from among other related interfering compound in skin due to its unique ability to 'fingerprint' molecules with long conjugates double-bond structure. With low-energy laser as a light source at wavelength $470 \mathrm{~nm}-490 \mathrm{~nm}$ which directed onto the surface of various anatomical skin sites such as inner palm, Raman resonance light scattering cause the carotenoids to emit a green signal at 510-530 nm which is quantified and detected. Thus the Raman scattering method shows as a non-invasive measurement of skin carotenoids [30], [31]. Investigations of pigmentation and UV photosensitivity also have been done to show the significant role of carotenoids in human skin. Consequently, tissue tends to faced more challenging in optical scenario such as highly heterogeneous, variety of chromophores that simultaneously excited with carotenoids and generate fluorescence thus poses difficulty in identifying Raman signal. One of the solutions is in selected anatomical skin site with low of pigmentation or melamine, thick stratum corneum and using two excitation sources in sequential manner.

Carotenoids detection of invasive HPLC technique is not a real-time system while detection using non-invasive Raman spectroscopy especially for skin carotenoids is a real-time system where respond to a signal, event or request fast enough to satisfy some requirement in substances identification and measurement.

Basic components of Raman spectroscopy consists of excitation source, sample illumination system and light collection optics, filter and detector. Recently, some of the skin carotenoids using LEDs (light emitting diode) instead of laser as light source because of some favorable characteristic: (a) good light source because of small in size (b) low cost or inexpensive (c) easy to handle and drive because used simple power supply and (d) emit in a near monochromatic light [32]. LED-based instrument is less involved in safety regulation compared to some regulation deal if work with laser-based instrument. This is because the basic characteristic of LED which have very limited radiance and do not constitute a clear eye hazard. Study has 
show the skin carotenoids measurement using LED as excitation source is high in sensitivity, easy to rebuild and produce high throughput. This approach also offers size and cost reduction and the problem of high tolerance effect in thermal of a laser-based Raman has been overcome [33].

\section{FUtURE TREND IN CAROTENOIDS DETECTION INVESTIGATION}

The trends of types of carotenoids detection can be represent in Fig. 3 where it shows the number of publication ranging from year 1995 until 2010 in a various type of detection via plasma, retina, skin and others. We agree that by collecting and analyzing the number of publication of related area throughout the year will give some general information on prediction of the trends in a future. By isolating the group of types of detection of specific area especially in human would give some guideline in constructing and generate a new idea in a future for the importance of the study.

The number of publication listed is collected from the source of Web of Science homepage which provide analyzed tools in order to reclaim precise citation counts.

From Fig. 3, plasma carotenoids seem to increase rapidly and lead with a total number of publication approximately 1248 publications. This is because plasma carotenoids always perform as standard biomarker and validation tools for all types of detection using high invasive gold standard method of HPLC. For skin carotenoids, before year 2000, the publication is low and small. Nevertheless, by the year 2000 and above, detection through skin carotenoids increase with a total number approximately 422 publications and this is because of development of non-invasive Raman spectroscopy technique. Non-invasive Raman spectroscopy give an alternative where offer a rapid, fast, high sensitive and high accuracy result. Although HPLC technique is a gold standard technique but for skin carotenoids the technique is not suitable and practical because of large tissue sample is needed and requires biopsies during the process.

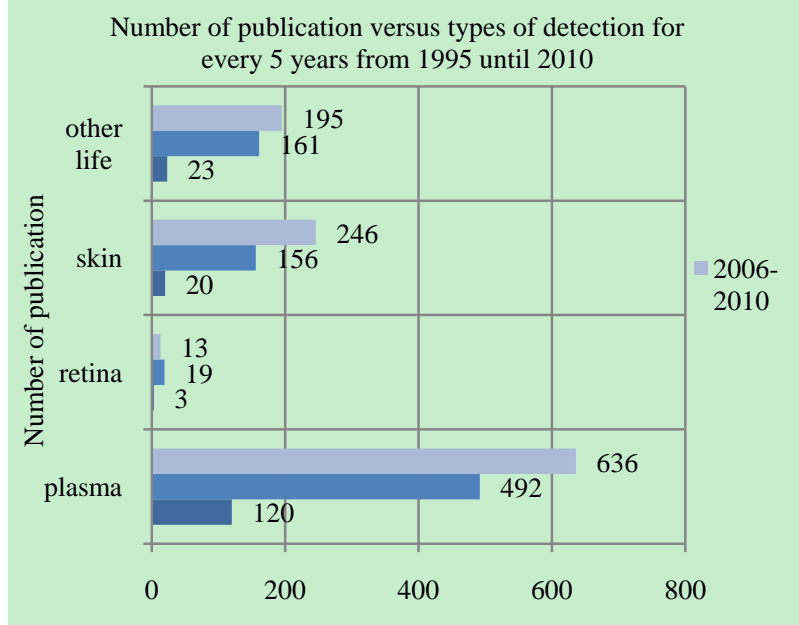

Fig. 3. Histogram of number of publication versus types of carotenoids detection: plasma, retina, skin and other life from 1995 until 2010.

During early years from 1995 until 2000, detection of carotenoids via macula pigment of retina is very low because of lack and difficulty in getting eye donor for data collection but from year 2001 and above the trend of macula pigment of retina increased because the awareness of researcher in the risk of eye disease especially in AMD disease and development of non-invasive technique with a total publications of 35 . With a total publication of 379 , carotenoids detection among animal such as marine life, mammals, birds, vertebra and others increase rapidly and getting popular among researcher recently. The trends is keep increasing among various researcher activities with many types of animal are associated with carotenoids content and more area can be explore such as to prove the presence of carotenoids as well as their function or role in their life.

The graph plotted in Fig. 4 shows the linear equations that generate the similar data from Fig. 3.

The expression is presented for each detection area from a standard linear expression of $y=m x+c$ where $m$ equal to the gradient or slope of the line to examine the overall potential of changes over time. The prediction value of a number of publications for 2011 until 2015 was calculated by the following equation:

Plasma carotenoid,

$$
y=258 x-100
$$

Skin carotenoid,

$$
y=113 x-85
$$

Retina carotenoid,

$$
y=5 x+2
$$

Other life carotenoids,

$$
y=86 x-46
$$

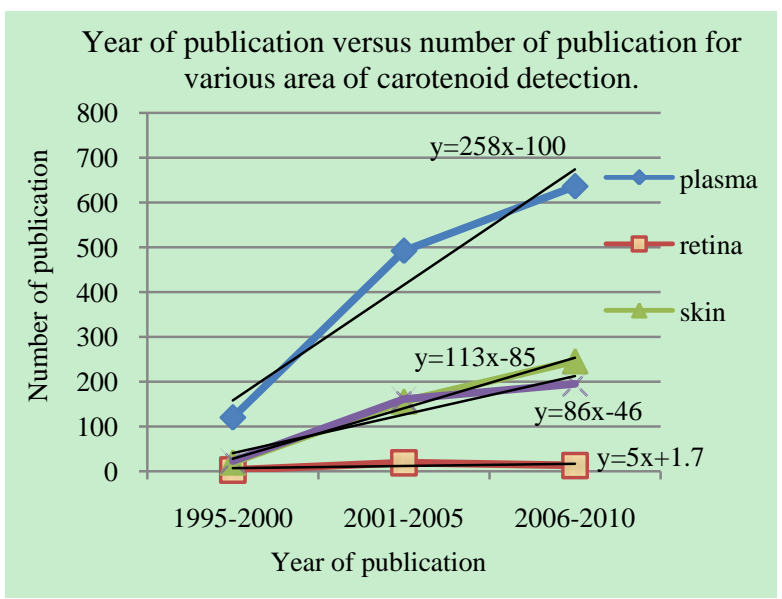

Fig. 4. Graph representing the types of carotenoids detection with a number of publications from 1995 until 2010.

From expression 1-4, the slope of plasma carotenoids is the highest among the other types of carotenoids detection. The variable $y$ represents the number of publication while variable $x$ represent the years. The expressions indicate that how much the number of publication changes for each unit of change in every 5 years. We can see the proportional of relationship between the numbers of publication with year of publication. Based on the previous and current data, indicate that there are a continuously trend in carotenoids 
detection and getting higher from year to year. The prediction values of publication have been presented over a period of time in a plotted graph in Fig. 5.

From Fig. 5, we can conclude that most of the carotenoids detection area still has high interest among researcher although there is likely almost saturate for macula retina detection. With the increasing of publication through all types of detection, the plasma seems to have the large number of publication. This is because, most of the researcher found plasma carotenoids is suitable in validate their finding and data. Through HPLC techniques, carotenoids detection via plasma became an important method in order to confirm the experimental result. As a biomarker, carotenoids detection through plasma is predicted to rapidly increase for another 5 years as well as detection via skin and other life.

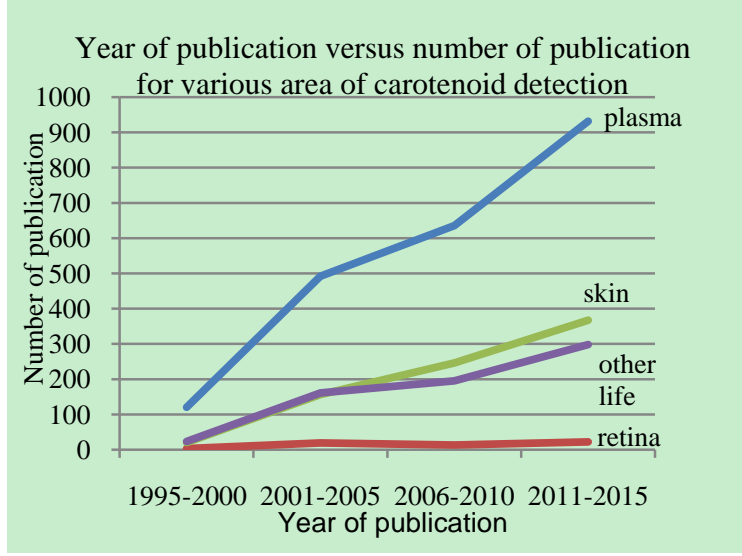

Fig. 5. Prediction graph representing the types of carotenoids detection with a number of publications from 1995 until 2015

Prediction of carotenoids detection through the number of publication give a direction in determining the area of interest in order to improve the impotency of carotenoids itself in our life. In addition, research activities will continuously raise as a research of carotenoids interest is increased. We can see that direction of carotenoids detection in a future as a guideline in planning the related studies for more identification of carotenoids activities in human, animal, plants and others.

\section{CONCLUSION}

As a conclusion, we directly predict the future trends of carotenoids detection and we observed that the trends of detection carotenoids are significant with the number of publication. This is because the development of various detection methods and techniques grows rapidly. The carotenoids detection technique can be divided into two groups: invasive and non-invasive methods. Highly invasive technique of HPLC as gold standard method is widely used as a verification tools and biomarker for fruits and vegetable intake. Thus, Resonance Raman spectroscopy appears to be non-invasive technique which offers high accuracy, sensitivity, practical and rapid methods especially when processing a large tissue volume. With a development of alternative carotenoids detection methods, information on carotenoids role, characteristic and its behaviour in human including animals and plants will widely explore and investigate among epidemiology and research activities.
In a future, more detection area of carotenoids may be explore with a various selection of invasive or non-invasive technique in measuring antioxidant status as preventing agent from chronic disease and carotenoids role in human and non-human respectively. This research have benefits to plan the direction of carotenoids detection in research activities in order to enhance role of carotenoids as an essential study to helped increase the visibility of carotenoids detection issues and create the new field of the related study. It is also as a guideline and motivation for a researcher to concentrate and focused on which area which give most beneficial information especially in developing strategies for management, treatment and prevention of high risk disease.

\section{ACKNOWLEDGEMENT}

The present study support by Arus Perdana-ICT grant, Universiti Kebangsaan Malaysia.

\section{REFERENCES}

[1] M. E. Kerr, C. M. Bender, and E. J. Monti, "An introduction to oxygen free radicals," Heart Lung, vol. 25, pp. 200-209, 1996.

[2] W. Stanhl et al., "Biological activities of natural and synthetic carotenoids induction of gap junctional communication and singlet oxygen quenching," Carcinogenesis, vol. 18, no. 1, pp. 89-92, 1997.

[3] B. Halliwell, "Free radicals, antioxidants and human disease curiosity, cause or consequence?" The Lancet, vol. 344, pp. 721-724, 1994.

[4] G. Britton, "Structure and properties of carotenoids in relation to function," FASEB J., vol. 9, pp. 1551-1558, 1995.

[5] N. I. Krinsky and E. J. Johnson, "Carotenoid actions and their relation to health and disease," Molecular Aspects of Medicine, vol. 26, pp. 459-516, 2005.

[6] D. Dutta, U. R. Chaudhuri, and R. Chakraborty, "Structure, health benefits, antioxidant property and processing and storage of carotenoids," Africal Journal of Biotechnology, vol. 4, no. 13, pp. 1510-1520, 2005.

[7] J. M. Seddon, U. A. Ajani, and R. D. Sperduto, "Dietary carotenoids, vitamins A, C, and E, and advanced age-related macular degeneration," JAMA, vol. 272, pp. 1413-1420, 1994.

[8] M. E. Darwin et al., "Non-invasive in vivo determination of the carotenoids beta-carotene and lycopene concentrations in the human skin using the Raman spectroscopic method. Journal of Physics D," Applied Physics, vol. 38, pp. 2696-2700, 2005.

[9] W. C. Willett et al., "Validation of a dietary questionnaire with plasma carotenoid and $\alpha$-tocopherol levels," American Journal of Clinical Nutrition, vol. 38, pp. 631-639, 1983.

[10] E. D. Brown et al., "Plasma carotenoids in normal men after a single ingestion of vegetables or purified $\beta$-carotene," American Clinical Nutritional Journal, vol. 49, pp. 1258-1256, 1989.

[11] M. M. Mathew-Roth, "Plasma concentrations of carotenoid after large doses of $\beta$-carotene," American Clinical Nutritional Journal, vol. 52, pp. 500-501, 1990

[12] K. L. Tucker et al., "Carotenoid intakes, assessed by dietary questionnaire, are associated with plasma carotenoid concentrations in an elderly population," Journal of Nutrition, vol. 129, pp. 438-445, 1999.

[13] R. A. Bone, J. T. Landru, and S. L. Tarsis, "Preliminary identification of the human macular pigment," Vision Rrs., vol. 25, no. 11, pp. 1531-1535, 1985.

[14] H. Cena, A. M. Castellazzi, and A. Pietri, "Lutein concentration in human milk during early lactation and its relationship with dietary lutein intake," Public Health Nutrition, vol. 12, pp. 1878-1884, 2009.

[15] T. TJM Berendschota and D. V. Norrena, "On the age dependency of the macular pigment optical density," Experimental Eye Research, vol. 81, pp. 602-609, 2005.

[16] P. S. Bernstein et al., "Raman detection of macular carotenoid pigments in intact human retina," Invest Ophthalmol Vis Sci., vol. 39, pp. 2003-2011, 1998.

[17] C. R. Gale et al., "Lutein and zeaxanthin status and risk of age-related macular degeneration," Invest Ophthalmol Vis Sci., vol. 44, pp. 24612465, 2003

[18] S. Alaluf et al., "Dietary Carotenoids contribute to normal human skin colour and UV Photosensitivity," Journal of Nutrition, vol. 132, pp. 399-403, 2002. 
[19] R. Lee et al., "The detection of carotenoid pigments in human skin," Journal of Investigation Dermatology, vol. 64, pp. 175-177, 1975.

[20] J. D. Ribaya-Mercado et al., "Skin lycopene is destroyed preferentially over beta carotene during ultraviolet irradiation in humans," Journal of Nutrition, vol. 125, pp. 1854-1859, 1995.

[21] I. V. Ermakov, M. R. Ermakov, and R. W. McClane, "Resonance Raman detection of carotenoids antioxidants in living human tissues," Optical society of America, vol. 26, pp. 1179-1181, 2001.

[22] H. Rajasingh, L. Oyehaug, D. I.e Vage, and S. W. Omholt, "Carotenoid dynamics in Atlantic salmon," BMC Biology, vol. 4, no. 10,2006

[23] S. Chatzifotis, I. Juan, P. Kyriazi, and M. Pavlidis, "Dietary carotenoids and skin melanin content influence the coloration of farmed red porgy (Pagrus)," Aquaculture Nutrition, vol. 29, September 2009, doi:10.1111/j.1365-2095.2009.00738.

[24] H. H. Aung et al., "Effect of dietary carotenoids on mouse lung genomic profiles and their modulatory effects on short-term cigarette smoke exposure," Genes Nutr., vol. 4, pp. 23-39, 2009.

[25] S. K. Clinton, "Tomatoes or Lycopene: A role in prostate carcinogenesis," Journal of Nutrition, vol. 135, pp. 2057-2059, 2005.

[26] F. Khachik, G. R. Beecher, and M. B. Goli, "Separation, identification and quantification of carotenoids in fruits, vegetables and human plasma by high performance liquid chromatography," Pure \& Appl. Chem., vol. 63, pp. 71-80, 1991.

[27] P. Bhosale, D. Y. Zhao, and P. S. Bernstein, "HPLC measurement of ocular carotenoid levels in human donor eyes in the lutein supplementation era," Investigative Ophthalmology \& Visual Science, vol. 48, pp. 543-549, 2007.

[28] I. V. Ermakov, M. R. Ermakov, and W. Gellermann, "Simple raman instrument for in vivo detection of macular pigments," Society for Applied Spectroscopy, vol. 59, no. 7, pp. 861-867, 2005
[29] I. Ermakov, M. Ermakov, and W. Gellermann, "Macular pigment Raman detector for clinical application," Journal of Biomedical Optics, vol. 9, pp. 139-148, 2004

[30] T. R. Hata et al., "Non invasive Raman spectroscopic detection of carotenoids in human skin," Journal of Invest Dermatology, vol. 115, pp. $441-448,2000$

[31] I. V. Ermakov, M. R. Ermakova, and W. Gellermann, "Noninvasive selective detection of lycopene and beta carotene in human skin using Raman spectroscopy," Journal of Biomedical Optics, vol. 9, pp. 332$338,2004$.

[32] K. Neelam et al., "Measurement of macular pigment: Raman spectroscopy versus heterochromatic flicker photometry," Investigative Ophthalmology \& Visual Science, vol. 46, pp. 3, 2005.

[33] S. D. Bergeson et al., "Resonance Raman measurements of carotenoids using light emitting diodes," Journal of Biomedical Optics, vol. 46, pp. 1023-1032, 2008

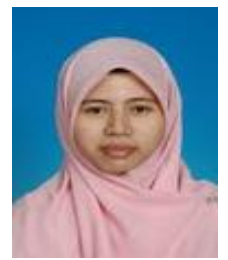

Rosdina Rahiman was born in Johor, Malaysia on 5th March 1975. She obtained her bachelor hons of electrical engineering (microelectronics) from UTM in 1998 and in 2002 earned his master in electrical engineering (MEE), also from the same institution. Major in electrical engineering of electronics, communication and biomedical area.

She works as a lecturer at UTM Razak School of Engineering and Advanced Technology, UTM Inter-national Campus, KL Malaysia. She is currently a $\mathrm{Ph}$. D. student under the supervision of Prof. Dr Mohd alauddin Mohd Ali at Universiti Kebangsaan Malaysia. Her research is centered on development of instrumentation of non-invasive carotenoids skin detection in human skin. 